\title{
Splenic infarction: An uncommon presentation of acute infectious mononucleosis
}

Madhulika Urella, MD ${ }^{1}$, Roma Srivastava, $\mathrm{MD}^{1}$, Waseem Ahmed, MD², Yehuda Lebowicz, $\mathrm{MD}^{1}$

\section{Author Affiliations:}

1. Joan C. Edwards School of Medicine, Marshall University, Huntington, West Virginia

2. Marshall University, Huntington, West Virginia

The authors have no financial disclosures to declare and no conflicts of interest to report.

\section{Corresponding Author:}

Madhulika Urella, MD

Joan C. Edwards School of Medicine

Marshall University

Huntington, West Virginia

Email: madhulika.urella@gmail.com 


\section{Abstract}

Splenic infarction is a relatively uncommon diagnosis. It occurs when the splenic artery or one of its sub-branches is occluded with an infected or bland embolus or clot. Splenic infarction may be caused by atrial fibrillation, bacterial endocarditis, sickle cell disease, antiphospholipid syndrome, and trauma whereas an infectious etiology is uncommon. It is considered a rare presentation of acute infectious mononucleosis. Currently, its pathogenesis is still unclear. We describe a 24-year-old African American female who was admitted for evaluation of left-sided chest pain. Chest imaging, an abdominal ultrasound, and initial laboratory data were normal, followed by a negative hypercoagulability panel. Signs and symptoms of infectious etiology were absent, however, both IgM and IgG antibodies for Ebstein-Barr virus (EBV) viral capsid antigen were high. Contrast-enhanced abdominal computed tomography revealed splenomegaly and multiple infarcts in the spleen, which eventually led to the diagnosis of infectious mononucleosis-associated splenic infarction, resolving the diagnostic dilemma.

\section{Keywords}

Epstein-Barr virus, infectious mononucleosis, splenic infarction

\section{Introduction}

Splenic infarction is a rare presentation of acute infectious mononucleosis, occurring when the splenic artery or a sub-branch becomes occluded with an embolus or clot. It may be caused by an intracardiac thrombus, hypercoagulable state, or splenomegaly whereas an infectious etiology is uncommon.

\section{Case Presentation}

A 24-year-old African American female with a known past medical history of type 2 diabetes mellitus, anxiety, insomnia, and morbid obesity presented to the emergency room with a threeday history of left-sided chest pain, nausea, and vomiting. She described her pain as sharp, 9/10 in intensity, continuous, and radiating to her left shoulder; the pain was aggravated upon deep inspiration. She was a current and longtime smoker at the time of admission. The urine drug screen was positive for cannabis. The only significant family history was a provoked pulmonary embolism in her mother, which occurred after a surgical procedure. The only pertinent physical examination finding was bilateral tenderness on deep palpation of the abdomen, worst in the left upper quadrant (LUQ). She was admitted for evaluation and supportive treatment.

The laboratory data was significant for thrombocytopenia, neutropenia, lymphocytosis, and normocytic anemia. Lactate dehydrogenase was elevated and liver function tests were within normal limits. The peripheral blood smear was normal, except for atypical lymphocytosis and some enlarged platelets. Chest imaging and abdominal ultrasound of the right upper quadrant with Doppler flow were both normal. At this point a hematology consult was requested, resulting in hemoglobinopathies being ruled out and a negative hypercoagulability panel. The patient's hospital course was complicated by a fever, prompting a monospot test which turned out to be negative. Blood cultures, thyroid function tests, rheumatoid factor, serology for HIV, hepatitis and other infections, electrocardiogram, and urinalysis all showed normal findings. The presence of high levels of IgM and IgG antibodies directed against the Ebstein-Barr virus viral capsid antigen (VCA) as well as contrast-enhanced computed tomography (CT) of the abdomen 
exhibiting multiple splenic infarcts and splenomegaly, with spleen measuring $17.5 \mathrm{~cm}$ in length, (Figure 2) led to the rare diagnosis of splenic infarction secondary to acute infectious mononucleosis. The hospital course was uneventful and she was discharged after one week with advice to take rest at home. IgM antibodies waned during her follow-up visit whereas IgG antibodies still persisted. Although the patient did not show any prior signs and symptoms of primary EBV infection, she presented with splenic infarction which is one of the rare complications of acute infectious mononucleosis.

Figure 1: Contrast enhanced CT demonstrating a splenic infarct

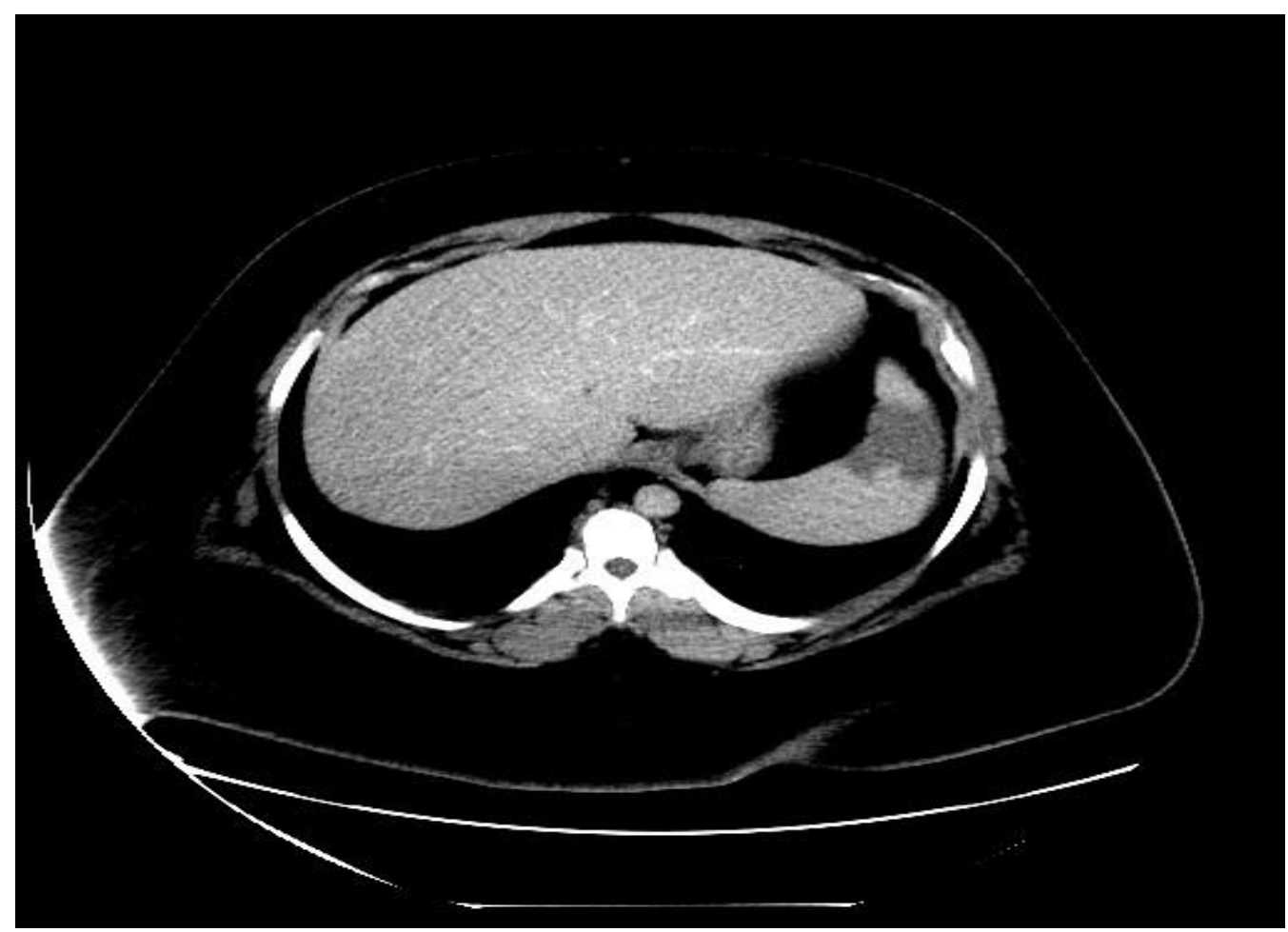


Figure 2: Contrast enhanced CT showing multiple splenic infarcts

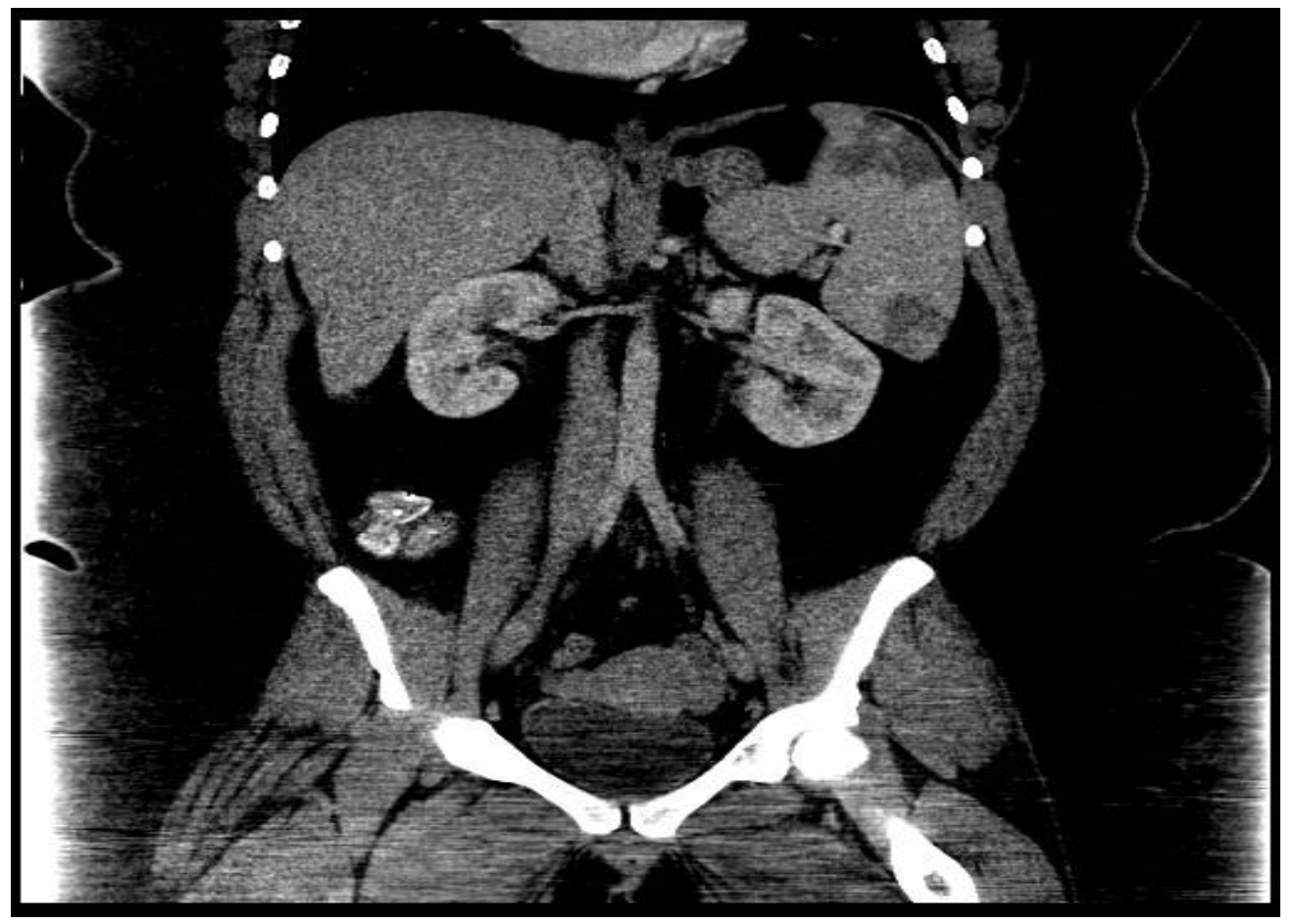

Figure 3: Contrast enhanced CT showing wedge shaped area of splenic infarct

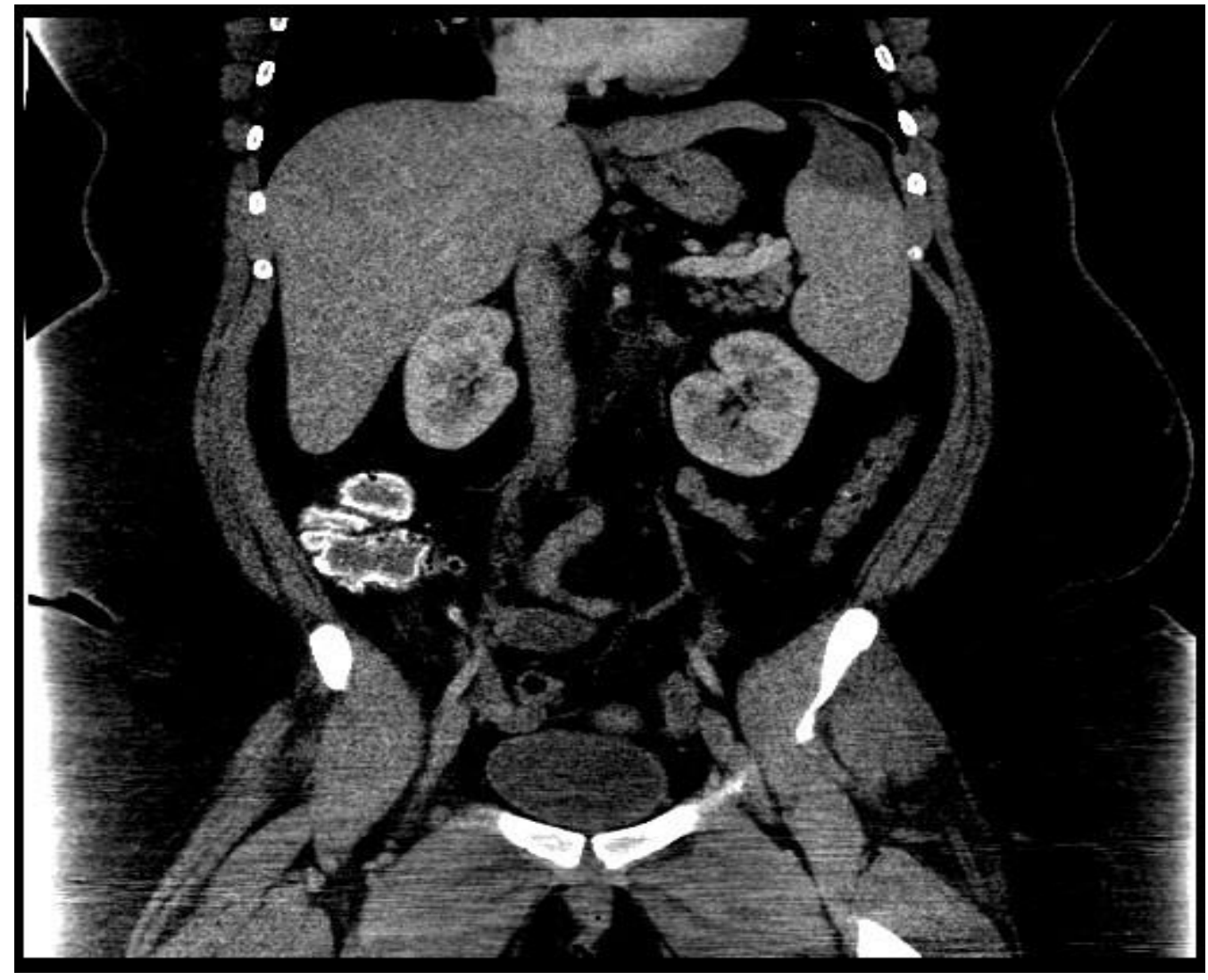




\section{Discussion}

Splenic infarction occurs when the splenic artery or one or more of its sub-branches become occluded with an infected or bland embolus or clot. This is a rare diagnosis, with only 19 cases reported in the literature at the time this manuscript was published; hence the exact incidence is unknown. A major study was conducted over a period of 11 years which revealed that $71 \%$ of the patients with splenic infarcts had predisposing factors. Atrial fibrillation was the most common predisposing factor, found to be in $23 \%$ of the patients, while EBV infection was diagnosed in $4 \%$ of patients. Interestingly, in $16.6 \%$ of the patients, the diagnosis of splenic infarction led to the further identification of serious underlying conditions such as a hypercoagulable state, atrial fibrillation, and polycythemia vera. ${ }^{1}$

The exact pathogenesis of splenic infarction is still not completely understood. Thromboembolism and massive enlargement of the spleen are considered the major causes for splenic infarction. ${ }^{1}$ Thromboembolism could result from an intracardiac thrombus or as the result of a hypercoagulable state. ${ }^{2,3}$ Review of the literature revealed splenomegaly as the predisposing factor in $10 \%$ of patients, which could be the result of an acute infection, as in our case, or it could be the result of malignant or non-malignant hematologic disease. ${ }^{1,2,4,5,6}$ Splenomegaly leads to alteration of the splenic microcirculation, which in turn effects splenic function. Several cases of infectious mononucleosis-associated splenic infarction have been reported to be associated with preexisting causes of a hypercoagulable state, such as antiphospholipid syndrome, sickle cell disease, spherocytosis, and protein $\mathrm{C}$ and protein $\mathrm{S}$ deficiency. ${ }^{2,7,8}$

The clinical presentation of splenic infarction may vary from asymptomatic to a fatal subcapsular hemorrhage. In one literature review, among all splenic infarcts reported until now, 20-30\% were reported to be asymptomatic. ${ }^{9,10}$ Almost $80 \%$ of patients presented to emergency room with leftsided abdominal pain. ${ }^{1}$ The most frequent symptom was LUQ pain, with LUQ abdominal tenderness in two-thirds of patients. ${ }^{1,10}$ Other signs and symptoms include fever, vomiting, splenomegaly, pleuritic chest pain, and pain referred to the left shoulder (Kehr's sign). In laboratory data, elevation of lactate dehydrogenase $(\mathrm{LDH})$ was reported in $69 \%$ of the patients. ${ }^{1}$

Contrast-enhanced CT scan is currently the best imaging modality available to diagnose splenic infarctions. It demonstrates the size of the spleen and peripheral wedge-shaped regions of hypoattenuation within the spleen. Ultrasonography has a very low diagnostic yield for acute splenic infarction, with sensitivity reported to be only $18 \%{ }^{1,9}$ Still, it can be useful in the followup of patients with acute splenic infarction and for the detection of possible complications, including subcapsular or peritoneal hemorrhage, pseudocyst, and abscess formation. ${ }^{10}$ Almost all of the cases of infectious mononucleosis-associated splenic infarction have been managed conservatively, with no inpatient hospital mortality. Close observation is still required though, as reinfarction could result in splenic rupture. ${ }^{10}$

\section{Conclusion}

Splenic infarction is a rare complication of acute infectious mononucleosis. ${ }^{1}$ The absence of classic signs and symptoms of infection prompts a high index of suspicion for this condition, as it has been the presenting symptom of serious underlying diseases. Our case specifically portrays splenic infarction in the absence of predisposing factors, which is an exceptional presentation. 
Therefore, splenic infarction requires attention from the medical fraternity as one of the possible presentations of acute infectious mononucleosis. 


\section{References}

1. Antopolsky M, Hiller N, Salameh S. Splenic infarction: 10 years of experience. Am J Emerg Med 2009; 27(3):262-5.

2. Van Hal S, Senanayake S, Hardiman R. Splenic infarction due to transient antiphospholipid induced by acute Epstein-Barr virus infection. J Clin Virol 2005; 32(3):245-7.

3. Tan DC, Low AH, Ong HS. Unusual abdominal manifestations of catastrophic antiphospholipid syndrome. Br J Haematol 2006; 132(5):538.

4. Symeonidis A, Papakonstantinou C, Seimeni U. Non hypoxia related splenic infarct in a patient with sickle cell trait and infectious mononucleosis. Acta Haematol 2001; 105(1):53-6.

5. Beckett D, Miller C, Ferrando JR. Case report: polycythaemia vera presenting as massive splenic infarction and liquefaction. Br J Radiol 2004; 77(922):876-7.

6. Suzuki Y, Shichishima T, Mukae M. Splenic infarction after Epstein- Barr virus infection in a patient with hereditary spherocytosis. Int J Hematol 2007; 85(5):380-3.

7. Gavriilaki E, Sabanis N, Paschou E. Splenic infarction as a rare complication of infectious mononucleosis due to Epstein- Barr virus infection in a patient with no significant comorbidity: case report and review of the literature. Scand J Infect Dis 2013; 45(11):888-90.

8. Gang MH, Kim JY. Splenic infarction in a child with primary Epstein-Barr virus infection. Pediatr Int 2013; 55(5):e126-8.

9. Nores M, Phillips EH, Morgenstern L. The clinical spectrum of splenic infarction. Am Surg 1998; 64(2):182-8.

10. Jaroch MT, Broughan TA, Hermann RE. The natural history of splenic infarction. Surgery 1986; 100(4):743-50. 